\title{
Pertinencia laboral y social del programa de Tecnologia en Regencia de Farmacia en la región de Urabá
}

\author{
Work and social relevance of the Regency of Pharmacy \\ technology program in the Urabá region
}

Olga L Arroyave G', William A Álvarez V V , Pedro Amariles ${ }^{1}$, Martha G Vásquez Z ${ }^{1}$, Beatriz E Cardona $\mathrm{Y}^{1}$

Forma de citar: Arroyave O, Álvarez W, Ameriles P, Vásquez M, Cardona B. Pertinencia laboral y social del progrma de Tecnología en Regencia de Farmacia en la región de Urabá. Rev Univ Ind Santander Salud. 2016; 48(4): 526-534. DOI: http:// dx.doi.org/10.18273/revsal.v48n4-2016011 @c) (1)

\section{RESUMEN}

Introducción: La Universidad de Antioquia desarrolla, en el Urabá antioqueño, el programa de Tecnología en Regencia de Farmacia (TRF), orientado a formar en promoción de la salud y prevención de la enfermedad. Objetivo: Caracterizar la pertinencia social y laboral del programa de TRF en la región de Urabá, y su efecto en la promoción de la salud y prevención de la enfermedad. Materiales y métodos: Estudio descriptivo de corte trasversal, en el cual se diseñaron cuatro instrumentos, orientados a egresados, empleadores, establecimientos y comunidad de la región, para ser aplicados por estudiantes del programa en la región, durante un periodo de seis meses. Resultados: De 52 egresados encuestados, 51 eran de la región, y 48 de ellos estaban laborando, 42 en la región. Los empleadores valoraron los aportes del TRF, como bueno y excelente, en un 69.6\%, 78.2\% y 78.3\%, para los desempeños institucional, procesos administrativos y asistenciales, respectivamente. En cuanto a la comunidad, 212 de los 369 encuestados, identificaron la existencia del TRF en farmacias/droguerías y esperan que le brinde información sobre el uso de los medicamentos. La pertinencia social fue valorada como pertinente y muy pertinente, en un $77.4 \%$, resultado que fue similar para la pertinencia laboral. Conclusiones: El estudio identifica un alto de grado de satisfacción con las funciones del TRF en los servicios farmacéuticos y de aceptación e identificación del TRF por la comunidad. El programa de TRF es pertinente en la región, sus egresados están ubicados en la región, desempeñando funciones acordes con su formación.

Palabras clave: Social, Laboral, Servicio Farmacéutico, Comunidad, Graduados, Farmacia (DeCS).

\begin{abstract}
Introduction: University of Antioquia develops, in the Urabá region of Antioquia (Colombia), the program of Technology in Regency of Pharmacy (TRP), aimed at training in health promotion and disease prevention. Objective: To characterize the social relevance of the TRP program in the Urabá region, and its effect in health promotion and disease prevention. Materials and methods: cross-sectional study with descriptive metodology, in which four instruments were designed and aimed at graduates, employers, institutions and community in the region, to be

1. Universidad de Antioquia. Medellín, Colombia

Correspondencia: Olga Lucia Arroyave G. Dirección: C11 17 A \# 75-11 Correo electrónico: olga.arroyave@udea.edu.co. Teléfono: 2195450.


applied by the students of the program in the region, for a period of six months. Results: Of 52 graduates surveyed, 51 were graduates of the region, and 48 of them were working, 42 in the region. Employers valued the contributions of the TRP, as good and excellent, in a $69.6 \%, 78.2 \%$ and $78.3 \%$, for institutional performance, administrative and welfare, respectively. Related to the community, 212 of the 369 respondents identified the existence of the TRP in pharmacies/drugstores and they expected that she/he would give you information about the use of drugs. The social relevance was assessed as relevant and very relevant, in a $77.4 \%$, a result that was similar to the relevance related to work. Conclusions: The study identifies a high degree of satisfaction with the functions of the TRP in pharmaceutical services and acceptance and identification of the TRF for the community. The TRP program is relevant in the region; its graduates are working in the region, performing duties commensurate with their training.

Keywords: social relevance, job relevance, graduates, employers, local, community, pharmacy regency (MeSH).

\section{INTRODUCCIÓN}

La Facultad de Ciencias Farmacéuticas y Alimentarias de la Universidad de Antioquia hace presencia en las regiones del departamento de Antioquia con programas académicos, de extensión y de investigación. En este contexto, la Facultad cuenta con el programa de Tecnología en Regencia de Farmacia (TRF), el cual se centra en formar en promoción de la salud y la prevención de la enfermedad en la comunidad, a través de su objeto de estudio (el medicamento), contribuyendo al sector farmacéutico del país.

El programa de TRF fue creado por la Facultad desde $1967^{1}$, cuyo perfil busca formar egresados integrales comprometidos con la sociedad, autónomos, libres y propositivos, con una formación en áreas básicas, administrativas y asistenciales, durante seis semestres académicos $^{2}$. La misión del programa es contribuir a la promoción de la salud y prevención de la enfermedad, en los ámbitos individual, familiar y colectivo, formando un talento humano íntegro, capacitado para la gestión, la vigilancia, el seguimiento y la auditoría del uso de los medicamentos en la farmacia ambulatoria y hospitalaria de baja complejidad y para el desarrollo de tareas de apoyo en la prestación del servicio farmacéutico de mediana y alta complejidad ${ }^{1-5}$.

Actualmente, la Facultad oferta el programa de TRF en las regiones: Urabá, Caucasia, Carmen del Viboral, Puerto Berrío, Yarumal, Santa Fe de Antioquia, Segovia, Andes, Amalfi y Envigado. Finalizando el 2014, el programa tenía cerca a los 1600 egresados, de los cuales, el $25 \%$ ha sido graduado en las regiones del departamento de Antioquia. En este sentido, Urabá es la región que presenta el mayor número de egresados, en cual la Facultad hace presencia desde 1995 y su primera cohorte de graduandos fue en 1999.

En el momento, la Facultad no dispone de información consolidada sobre el desempeño laboral, el efecto, el reconocimiento y la necesidad social del programa, en especial en las políticas de salud pública y el mejoramiento de la calidad de vida de la comunidad. Por ello, se ve necesario establecer, desde la perspectiva académica, el medio y su relación con los empleadores y egresados, la pertinencia social y laboral del programa académico en la región de Urabá antioqueño. Entendiendo pertinencia social como el conjunto de actividades que podría desarrollar, y para las cuales está capacitado el profesional en Tecnología en Regencia de Farmacia en el ámbito de la promoción de la salud y prevención de la enfermedad y su interacción con la comunidad; la pertinencia laboral como la captación y las condiciones de las mismas, que realizan los empleadores y las instituciones de la salud en la región del TRF. Con este soporte, se puede proyectar la valoración del efecto que dicho programa tiene en la regiones ${ }^{6}$. En este sentido, los objetivos del presente trabajo fueron: 1) caracterizar la pertinencia social y laboral del programa de TRF en la región de Urabá, y su efecto en la promoción de la salud y la prevención de la enfermedad; 2) caracterizar los servicios y/o establecimientos farmacéuticos de la región; y 3) determinar el grado de conocimiento de la comunidad en general sobre los aportes sociales y sanitarios del TRF.

\section{MATERIALES Y MÉTODOS}

\section{Tipo de estudio: Descriptivo, retrospectivo y de corte trasversal}

Instrumentos. La recolección de la información se fundamentó en la aplicación de instrumentos de medición cuantitativa (encuestas y bases de datos históricas), relacionada con el efecto, pertinencia y reconocimiento social. Se diseñaron cuatro instrumentos orientados a responder a los objetivos del proyecto así: 1) caracterización de los establecimientos y/o servicios farmacéuticos; 2) caracterización de los egresados; 3) valoración de la pertinencia laboral 
de acuerdo a los empleadores; y 4) valoración de la pertinencia social de acuerdo a la comunidad.

Prueba piloto y ajustes. Los cuatro instrumentos fueron revisados y ajustados, posteriormente, se realizó una prueba piloto para validar la claridad y funcionalidad de los instrumentos. Para ello, se aplicaron los instrumentos en otras sedes regionales, con el apoyo de estudiantes del programa de TRF. Los resultados de esta prueba mostraron la necesidad de mejorar la claridad y comprensión de algunas de las preguntas de los instrumentos direccionados a la comunidad y a los establecimientos farmacéuticos.

Muestra: En el caso de los egresados, se buscó una cobertura de los 113 egresados censados, al primer semestre 2014 de la región de Urabá. Con respecto a los establecimientos, según información proporcionado por la Dirección Seccional de Salud de Antioquia, al 2013 en la región existían 348 establecimientos registrados. Para la elección de la muestra, solo se tuvo en cuenta a los municipios con evidencia de la presencia de egresados del programa, realizando un muestreo aleatorio simple para una población finita con un nivel de confianza (95\% o $90 \%)$ y un error muestral deseado (5\%). En este sentido, se estimó una muestra de 183 establecimientos para un $95 \%$ de confianza y 152 con nivel de confianza del $90 \%$.

Relacionado con los empleadores y comunidades, la encuesta se aplicó, en los establecimientos, a los empleadores y usuario presentes al momento de aplicar el instrumento en el establecimiento. En el caso de la comunidad, se estimó encuestar a tres usuarios por establecimiento, representado en el Gráfico 1.

\section{Encuesta Egresado}

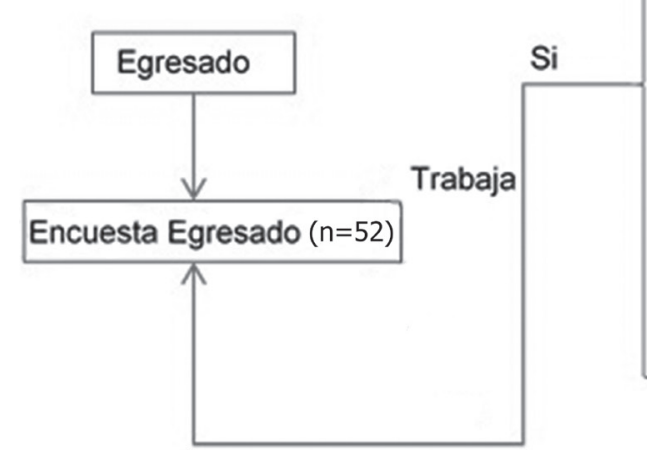

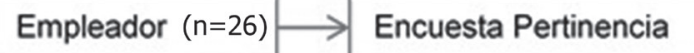

Establecimiento $(n=147) \rightarrow$ Encuesta Caracterización

Encuesta Caracterización

Comunidad $(\mathrm{n}=369) \rightarrow$ Encuesta Pertinencia

GRAFICO 1. Esquema para la selección de la muestra

Las encuestas con limitaciones en la información o mal diligenciadas fueron excluidas del análisis.

Análisis estadístico. La información se tabuló y procesó utilizado el programa estadístico SPSS versión 21 (SPSS Inc, Chicago, III). Se calculó la estadística descriptiva, incluyendo las proporciones y frecuencias. Con el fin de facilitar la presentación e interpretación de los resultados, las variables de tipo Likert, utilizando la técnica estadística de estratificación ${ }^{7}$, se agruparon en las categorías funciones asistenciales, administrativas y de desempeño laboral. Para el análisis del desempeño del TRF, según los empleadores, se utilizó la técnica de estratificación, con el fin de consolidar tres variables de desempeño (institucional, administrativa y asistencial) y el desempeño se evaluó como excelente, bueno o regular.
Acorde con la formación y experiencia del grupo de investigadores, para la valoración de la pertinencia social, se consideraron los resultados relacionados con la comunidad y con los empleadores. De forma detallada, para la comunidad se consideraron las variables reconocimiento del programa y las funciones del TRF en el servicio farmacéutico; por su parte del instrumento de los empleadores, se incluyeron las variables relacionadas con los procesos asistenciales, administrativas, mejoramiento del servicio y desempeño institucional. Acorde con la relevancia que determina la normatividad del servicio farmacéutico en Colombia ${ }^{3,5,8}$; a estas categorías se le asignaron los siguientes pesos porcentuales: 40, 30, 15 y $15 \%$, respectivamente y se establecieron las categorías: No Pertinente, Pertinente y Muy Pertinente. 
En el caso de la pertinencia laboral, se definió acorde con los resultados de las variables tiempo de vinculación, tipo de contrato y el nivel de ingresos de los egresados del estudio. Las cuales se agruparon en las mismas categorías definidas para la pertinencia social.

Consideraciones éticas: Se consideró una investigación sin riesgo, puesto que los métodos y estrategias no involucraron la intervención o modificación intencionada de variables biológicas, fisiológicas o sociales de las personas que participaron en ella ${ }^{9}$.

\section{RESULTADOS}

\section{Egresados}

Durante los seis meses de recolección de la información, sólo se pudo encuestar a 52 de los 113 egresados $(46 \%$ del total de graduados del programa de TRF en esta región). De ellos, 51 (98.1\%) eran egresados de Urabá y 1 de una región diferente. De los 51 egresados de Urabá, $48(92 \%)$ se encuentran laborando actualmente, de ellos $42(88 \%)$ en la región, $41(85 \%)$ están vinculados de tiempo completo, 5 (10\%) tiempo parcial, con una estabilidad laboral superior al año de vinculación, correspondiente al 70\% (37) de la muestra.

Con respecto a las actividades que desempeña el egresado, se encontró que 21 (44\%) de ellos ejercen como Director Técnico del establecimiento y/o servicio farmacéutico, 7 (15\%) como auxiliar de farmacia y 4 $(8 \%)$ como propietario. Por otra parte, $12(25 \%)$ afirma desconocer cuál es su función central que cumple en el establecimiento. Con relación al promedio salarial, se encontró que 25 (53\%) devengan menos de dos salarios minímos mensuales legales vigentes (SMMLV) de Colombia, 17 (35\%) mas de dos SMMLV, y $6(13 \%)$ no respondieron.

Con relación a la satisfacción del egresado con la formación académica, 42 (82\%) de los egresados se encuentran satisfechos con su nivel de formación. Por su parte, a la pregunta de cómo percibe su aceptación por parte de la comunidad, 45 (86\%) egresados consideraron que sus funciones profesionales son bien aceptadas por la comunidad; además, $46(89 \%)$ de ellos, consideraron, entre muy buena y excelente, la aceptación laboral en las instituaciones en las que se desempeñan.

En cuanto al cambio en la calidad de vida (vivienda, condiciones económicas, acceso a los servicios de salud y recreación), en promedio, 27 (52\%) egresados afirman que sus condiciones de vida son muy buenas y al menos 14 (27\%) excelente. Tabla 1.

\section{Establecimientos}

De los 147 establecimientos encuestados, 35 (24\%) son establecimientos comerciales, de los cuales 31 $(89 \%)$ son almacenes de cadena y $4(11 \%)$ almacenes de grandes superficies. Por su parte, 92 (63\%) corresponden a servicios farmacéuticos independientes, y de estos 89 (97\%) son establecimientos minoristas y solo 3 (3\%) son mayoristas; 20 (14\%) son servicios farmacéuticos dependientes, de los cuales $2(10 \%)$ son de alta complejidad, $6(30 \%)$ de mediana y $12(60 \%)$ de baja.

Con relación al grado académico del propietario del establecimiento, $16(11 \%)$ eran egresados de TRF, $24(16 \%)$ eran expendedores de medicamentos y 33 (22\%) eran propietarios con un título diferente al área de la salud. Relacionado a si el propietario labora en el establecimiento, 66 (45\%) laboran en el establecimiento y $76(52 \%)$ no laboran en el mismo.

Dentro de los servicios que prestaba el establecimiento, 34 (23\%) prestan servicios de inyectología, 75 (51\%) tienen ventas de dispositivos médicos, 25 (17\%) prestan servicios o a Instituciones Prestadoras de Servicios de salud (IPS) o tiene convenio con IPS, $44(30 \%)$ tiene servicio a domicilio y 42 (29\%) prestan otros servicio como comercio, venta de comestibles, helados, entre otros.

Con respecto a la forma de adquisición de los productos, $86(49 \%)$ afirmaron que los adquieren de distribuidores mayoristas, 51 (29\%) se adquieren en los laboratorios y 20 (11\%) en cooperativas, y los restantes (11\%) corresponden a distribuidores minoristas y otros establecimientos farmacéuticos. Igualmente $97(60 \%)$ consideraron que el TRF es la persona idónea para estar a cargo de los servicios farmacéuticos, 20 (12\%) a los Químicos Farmacéuticos (QF) y el resto a otras profesiones. A la pregunta si contrataría un TRF para manejar el establecimiento, 117 (90\%) manifestaron que si lo harían, mientras que $10(8 \%)$ informaron que no lo harían.

\section{Empleadores}

Con relación a las actividades que realiza el TRF, el total de los empleadores determinó que el desempeño de un TRF es 26 (100\%) en dispensación, 22 (85\%) en almacenamiento y control de fechas de vencimiento, 21 $(81 \%)$ en control de la temperatura y el uso adecuado del medicamento, como las funciones más representativas. 
Para la valoración de los empleadores sobre los aportes del TRF a la institución, se utilizaron 23 de las 26 encuestas, debido a que tres de ellas presentaban un alto porcentaje de preguntas sin respuesta. Con el fin de analizar el desempeño del TRF, según los empleadores se utilizó la técnica de estratificación para consolidar tres variables de desempeño (Institucional, administrativa y asistencial) y se evaluó el desempeño en tres categorías (Excelente, Buena y Regular).

En la Tabla 1 se presenta la valoración de los empleadores de los aportes del TRF, al agrupar las valoraciones bueno y excelente, se obtuvo una valoración del 69.6\%
(16), 78.2\% (18) y 78.3\% (18) para el desempeño institucional, en procesos administrativos y en procesos asistenciales, respectivamente.

En la Tabla 1, se presenta la valoración del empleador relacionado con el efecto de la vinculación del TRF en el servicio farmacéutico (considerando variables como el aumento de clientes, de ventas, satisfacción en la atención al usuario, funciones administrativas y funciones técnicas del establecimiento). En este sentido, 20 (87\%) de los empleadores considera que la vinculación del TRF ha mejorado o ha mejorado mucho el servicio.

TABLA 1. Resultados de la cambio en las condiciones de calidad de vida de los egresados, aportes de los mismos a los establecimientos, valoración del mejoramiento de los servicios farmacéuticos desde el punto de vista de los empleadores y valoración de la pertinencia social y laboral del egresado de Tecnología en Regencia de Farmacia de la región de Urabá

Cambio en las condiciones de la calidad de vida, luego de graduarse como TRF, de los egresados encuestados en la region de Urabá $(n=52)$

\begin{tabular}{lcccc}
\hline Condiciones de Vida & Vivienda (\%) & Condiciones Económicas (\%) & Acceso Servicios de Salud (\%) & Recreación (\%) \\
\hline Excelente & 23 & 31 & 27 & 23 \\
Muy Bueno & 54 & 56 & 56 & 44 \\
Bueno & 13 & 12 & 2 & 25 \\
Deficiente & 4 & 2 & 4 & 6 \\
Muy deficiente & 2 & 0 & 0 & 0 \\
N.R & 4 & 0 & 2 & 6
\end{tabular}

Aportes del Tecnólogo en Regencia de Farmacia al establecimiento (n=26 empleadores)

\begin{tabular}{lccc}
\hline Evaluación & $\begin{array}{c}\text { Desempeño en la } \\
\text { Institución }\end{array}$ & $\begin{array}{c}\text { Desempeño en los Procesos } \\
\text { administrativos }\end{array}$ & Desempeño en los Procesos asistenciales \\
\hline Excelente & 34.8 & 30.4 & 26.1 \\
Bueno & 34.8 & 47.8 & 52.2 \\
Regular & 30.4 & 21.7 & 21.7 \\
\hline
\end{tabular}

Valoración del mejoramiento del servicio farmacéutico después de la contratación del TRF (n=26)

\begin{tabular}{ll}
\hline Ha mejorado mucho & 34.8 \\
Ha Mejorado & 52.2 \\
No ha mejorado & 13.0 \\
\hline
\end{tabular}

Valoración de la pertinencia de acuerdo a los empleadores $(\mathbf{n}=\mathbf{2 6})$

\begin{tabular}{lcc}
\hline & Pertinencia Social según los Empleadores (\%) & Pertinencia Laboral del Egresado (\%) \\
\hline Muy Pertinente & 30.4 & 43.8 \\
Pertinente & 47.8 & 33.3 \\
No Pertinente & 21.7 & 22.9 \\
\hline
\end{tabular}

\section{Comunidad}

A la pregunta, si conocía en qué tipo de establecimiento se encontraba, $117(48 \%)$ de las 369 personas informaron que se encontraban en una farmacia particular, 46 (13\%) en un servicio institucional, y 128 (35\%) no logró identificar el tipo de establecimiento en el que se encontraban. 
Al indagar si conocían la existencia del programa de TRF en Colombia y sobre las funciones de este tecnólogo en los servicios de salud, $212(87 \%)$ lo identificaron en farmacias/droguerías y 199 (82\%) esperan que les brinden información sobre el uso de los medicamentos. Al momento de la atención, 138 (57\%) usuarios manifestaron que no identifican si el TRF se encuentra o no en la farmacia.

En cuanto a los servicios brindados en la farmacia/ droguería, se observó que 179 (49\%) usuarios opinan que el servicio está relacionado con el uso adecuado de los medicamentos, 64 (17\%) han recibido orientación sobre su problema de salud y 53 (14\%) sobre todos los servicios que ofrece la farmacia/droguería.
En cuanto al tipo de información que la comunidad recibe en las farmacias/droguerías, se observó que 222 (60\%) usuarios afirmaron que recibieron información sobre cómo se deben tomar los medicamentos, 141 $(38 \%)$ sobre las horas en las cuales debe tomar los medicamentos y 128 (35\%) el tiempo que deben tomar los medicamentos, como los ítems a resaltar en cuanto a la información que se brinda en estos establecimientos Gráfico 2. Igualmente, la comunidad consideró la atención recibida en la farmacia/droguería entre buena $47(13 \%)$, muy buena $128(35 \%)$ y excelente $78(21 \%)$.

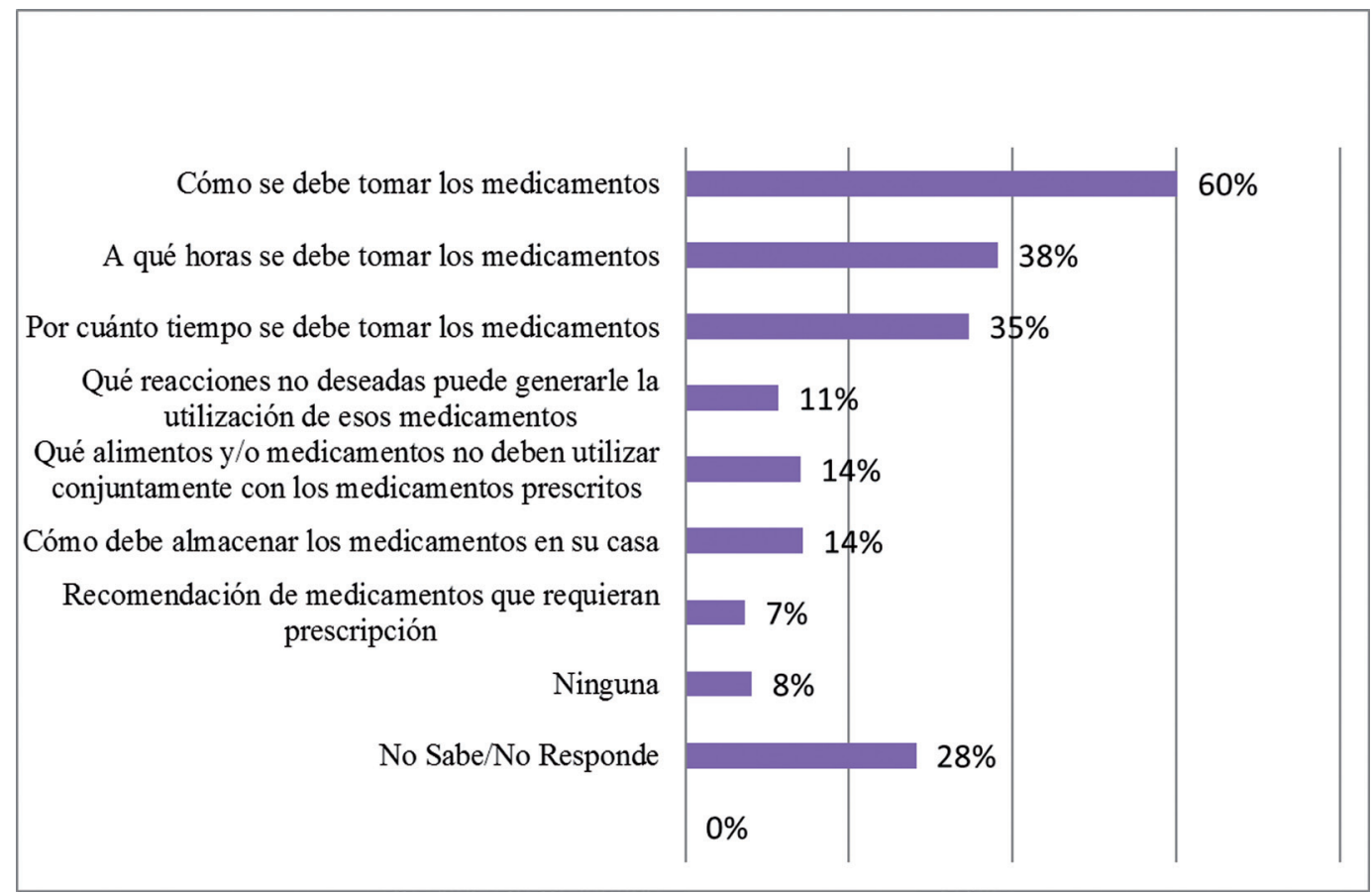

GRÁFICO 2. Tipo de información recibida en el servicio farmacéutico (n=369)

\section{Valoración de la Pertinencia}

\section{Pertinencia Social}

Para valorar la pertinencia social del TRF, la primera variable que se consideró fue el reconocimiento del programa por la comunidad. En este sentido, 243 (66\%) usuarios conocían sobre la existencia del programa de TRF y 126 (34\%) no conocía la existencia del mismo. A la pregunta si la comunidad considera que las funciones de una farmacia pueden ser ejercidas por cualquier tipo de profesional, se encontró que 228 (94\%) personas consideran que no puede ser cualquier profesional, 11 (5\%) consideraron que cualquier profesional puede ejercer las funciones de una farmacia y $4(2 \%)$ no 531 respondió. Por su parte, a la pregunta cuál considera que es la persona idónea para que lo atienda en una farmacia, $217(89 \%)$ consideraron que la atención la debe brindar un TRF, 59 (24\%) un QF, 24 (10\%) un Médico, 7 (3\%) una Enfermera. Por ello, en general, la labor del TRF fue reconocida por la comunidad.

Desde el punto de vista de los empleadores, la pertinencia del TRF se generó con la estratificación de los grupos definidos en el método. Para la distribución de esta variable se tuvo en cuenta los pesos porcentuales, acorde con la normatividad colombiana ${ }^{3}$. En este sentido, al desempeño asistencial se le dio un peso del $40 \%$, al desempeño administrativo un 30\%, al 
desempeño en la institución un $15 \%$ y al mejoramiento en el servicio un $15 \%$. De acuerdo a lo establecido en el método se establecieron tres categorías para la variable Pertinencia Social como No Pertinente, Pertinente y Muy Pertinente Tabla 1.

\section{Pertinencia Laboral}

La pertinencia laboral se construyó con las variables sobre tipo vinculación (Tiempo Completo, Tiempo parcial), Tipo de contratación (A término indefinido, A término definido, Provisional, Servicios, Honorarios) y sobre el nivel de ingresos (menos de un SMMLV, entre uno y dos SMMLV, entre dos y tres SMMLV y más de tres SMMLV), se encontró que la pertinencia laboral del egresado del programa de TRF es en un $43,8 \%$ es muy pertinente, $33.3 \%$ pertinente y $22.9 \%$ no pertinente Tabla 1.

\section{DISCUSIÓN}

La valoración de la pertinencia social y laboral del programa de TRF en la región de Urabá analizada en el estudio fue determinada por la percepción de la comunidad, por las funciones que desempeña en el servicio farmacéutico y por la ubicación laboral del mismo en la zona. Los resultados son de importancia para la Universidad y la oferta de sus programas en las regiones, pues con ello se reafirma su función social en esta zona.

Este estudio presenta diferencia con otros estudios similares, sobre pertinencia social y pertinencia laboral, debido a que los mismos se han centrado en valorar la calidad de los programas universitarios, como es el caso del programa de Ingeniería Química de la Universidad de Camagüey de Cuba, cuya evaluación se realizó teniendo en cuenta los parámetros de la acreditación de la carrera, dado que el criterio de pertinencia y el impacto social se encuentran incluidos como variable número uno en la guía de evaluación del SEA-CU ${ }^{10}$. El termino pertinencia, para la educación superior, en ocasiones se reduce a la respuesta que la misma da a las necesidades laborales o profesionales, sin embargo, Tünnermann Bernheim, considera que la pertinencia en la educación, debe estar ligada al papel de la educación en la sociedad y lo que está desea de ella ${ }^{11,12}$.

Las audiencias consideradas en el estudio, se determinaron acorde con los criterios de pertinencia, por esa razón los instrumentos fueron orientados a los egresados, los empleadores, los establecimientos y la comunidad. Teniendo solo en común con los otros estudios realizados, la valoración de los egresados, pero en cuanto a las características de valoración, si bien se abordan la calidad de la formación y el cambio en la calidad de vida del egresado, carecen de criterios como la vinculación, estabilidad laboral y el salario devengado, variables que son determinantes para la pertinencia laboral del estudio sobre el programa de TRF.

El concepto de pertinencia de la educación superior, es propuesto por la Unesco en 1998, para lo cual consideró que esta debe ser evaluada en función de lo que la sociedad espera de ella y lo que las instituciones realizan para dar respuestas a las necesidades de la misma ${ }^{13,14}$. En el estudio de la Universidad de Camagüey, se realizó una entrevista a los egresados, donde manifestaron el grado de satisfacción por su programa universitario y el desarrollo de competencias específicas de la profesión, destacando consideraciones éticas y morales ${ }^{10}$. Por su parte, otro estudio realizado en la carrera de Medicina de la Universidad de Pinar del Rio en Cuba, los egresados resaltan la influencia de su formación en la solución de problemas de su comunidad en su área del conocimiento $^{15}$.

Los resultados del presente estudio evidencian que la comunidad reconoce la idoneidad del TRF para el manejo de los servicios farmacéuticos; que existe una aceptación laboral del $70 \%$ por parte de los egresados; sin embargo, se identificó un porcentaje de los mismos que manifestó desconocer cuales son las funciones del TRF en el servicio farmacéutico. Por su parte, la limitación de la comunidad para la identificación del TRF en el establecimiento, puede indicar que, a pesar de la definición de competencias por parte de la instituciones de referencia a nivel nacional e internacional ${ }^{13}$ y las reformas curriculares el perfil de este egresado requiere afianzarse e interiorizarse de manera efectiva de forma tal, que tanto el empleador como la comunidad identifique el rol del TRF.

La academia, debe evaluar la percepción y valoración de los egresados por parte de la sociedad, lo que permite evidenciar si la Universidad como institución responde a las perspectivas y requerimientos sociales y de su propio entorno. Adicionalmente, la formación en las áreas de la salud debe responder, tanto a la necesidad tecnológica o como a la formación para el servicio ${ }^{13,16}$, por ello la dinámica de los procesos educativos debe dar cuenta de la pertinencia de los programas de formación acorde con las necesidades de la comunidad y del entorno laboral.

El presente estudio documenta la presencia de otros egresados universitarios en los establecimientos farmacéuticos, caso de administración de empresas, 
psicología y tecnólogos agropecuarios, quienes desarrollan diferentes funciones dentro de los establecimientos $\mathrm{y} / \mathrm{o}$ servicios farmacéuticos en la región. Este resultado podría indicar falta de identidad del TRF con su perfil y, por tanto, la Universidad debería diseñar estrategias para que el estudiante identifique su proyección profesional y su perfil laboral.

Los hallazgos de la valoración de la pertinencia social por parte del empleador, se encontró un alto de grado de satisfacción con las funciones del TRF en los servicios farmacéuticos, y de los cambios generados en los mismos a partir de la vinculación laboral del TRF. Históricamente a las Universidades se les ha atribuido la responsabilidad de las condiciones y cualidades de la captación de sus egresados en el mercado laboral, los objetivos de la formación y capacitación en educación, a la par que los programas académicos, se encuentran enfocados en este posicionamiento ${ }^{11,15,17}$.

Limitaciones: Estuvieron centradas en la recolección de la información la cual fue realizada por los estudiantes del programa en la misma región, se minimizó esta limitación con la capacitación por parte de los investigadores a los estudiantes y se brindó apoyo económico para el desplazamiento en la zona, igualmente los instrumentos no fueron validados, pero en su construcción se aplicó una prueba piloto en una región diferente y se revisó y corrigió posteriormente por el grupo de investigación.

\section{CONCLUSIONES}

Con relación a la ubicación laboral del TRF en la región, los resultados muestran que en el Urabá antioqueño, el programa de TRF es pertinente, sus egresados están ubicados en la región, desempeñando cargos para los cuales recibieron formación, y que las condiciones laborales, mediante las cuales se vinculan al mercado laboral, están acordes con su formación tecnológica. Igualmente, los resultados muestran que el programa es pertinente socialmente, aunque la comunidad no identifica totalmente al TRF en los servicios de farmacéuticos, si reconoce la importancia de sus funciones entorno el uso adecuado del medicamento, al igual que el empleador, en cuanto al mejoramiento y aumento de la calidad de estos establecimientos. Es importante para la Universidad y las asociaciones de egresados, en un trabajo conjunto diseñar estrategias para que la comunidad identifique más al TRF.

En la revisión bibliográfica realizada no se encontraron estudios sobre pertinencia laboral y social de un programa académico diseñados con este enfoque, como se enunció al inicio de la discusión, la valoración de la pertinencia está enfocada a la evaluación curricular de la calidad académica y la relación de la misma con el producto que entrega a la sociedad. Por ello, la propuesta metodológica para valorar la pertinencia laboral y social de este trabajo podría ser una referencia para otros estudios diseñados con este tipo de objetivos.

\section{AGRADECIMIENTOS}

A la Facultad de Ciencias Farmacéuticas y Alimentarias y al grupo de investigación de promoción y prevención por la financiación del proyecto, igualmente a los estudiantes del programa de la seccional Urabá los cuales apoyaron con la aplicación de los instrumentos.

\section{REFERENCIAS}

1. Universidad de Antioquia. [Internet]. Medellín: [Fecha de consulta 14 septiembre 2016]. Available: http://www.udea.edu.co/wps/portal/ udea/web/inicio/institucional/unidades-academicas/ facultades/ciencias-farmaceuticas-alimentarias/ ciencias-farmaceuticas-alimentarias

2. Universidad de Antioquia. Facultad de Ciencias Farmacéuticas y Alimentarias. Proyecto Educativo del Programa de Tecnología en Regencia de Farmacia. Universidad de Antioquia. 2005

3. Colombia. Ministerio de Educación. Ley 485 de 1998 por la cual se reglamenta la profesión Tecnólogo en Regencia de Farmacia. Diario Oficial, No. 43461, (Diciembre 29 1998).

4. Tobón FA, Gómez MC, Salamanca R. Responsabilidades en los actos farmacéuticos. Vitae 2001; 8 (1-2): 37-46.

5. Colombia. Ministerio de Protección Social. Decreto 2200 de 2005 por el cual se reglamenta el Servicio Farmacéutico. Diario Oficial 45954 (Junio 29-2005)

6. Navarro A, Álvarez M, Gottfried J. Pertinencia social de la Universidad. Una propuesta para la construcción de la imagen institucional. Educación Superior y Sociedad. 1997; 8 (2): 75-96.

7. Allen E, Seaman C. Likert Scales and Data Analyses. Quality Progress 2007; 40(7): 64-65.

8. Colombia. Ministerio de la Protección Social. . Resolución 1403 de 2007 por la cual se reglamenta el Modelo de Gestión del Servicio Farmacéutico. Diario Oficial No. 46.639 (Mayo 25-2007)

9. Colombia. Ministerio de Salud. Resolución 8430 de 1993, por la cual se establece las normas científicas, técnicas y administrativas para la investigación en salud. Bogotá: el Ministerio; 1993.

10. Julián M, Ugarte M, Portuondo R, Galindo P. 
Pertinencia e impacto social de la carrera de Ingeniería Química de la Universidad de Camagüey. Tecnología Química. 2005; 23 (3): 52-56.

11. Rojo N. Pertinencia social de los programas académicos de perfil gerencial en el sector salud. Rev Cubana Educ Med Sup 1999;13(1):70-79

12. Tünnermann, C. Pertinencia Social y Principios Básicos para orientar el diseño de Políticas de Educación Superior. Revista Educación Superior y Sociedad, 2000; 11 (1 y 2): 181-196.

13. UNESCO. Declaración Mundial sobre la educación superior en el siglo XXI: Visión y Acción. Conferencia Mundial sobre la Educación Superior. 1998. Disponible en: http://unesdoc.unesco.org/ images/0011/001163/116345s.pdf. [Fecha de consulta 10 de Abril de 2016].

14. Giordana P, Naidorf J, Horn M, La pertinencia social de la Universidad como categoría equívoca. Nómadas (Col) 2007 22-33.

15. Valdés A, Morales I, Díaz J, Sánchez A, Cuellar R. Criterios de evaluación de la variable "pertinencia e impacto social" específicos para la carrera de Medicina. Educación Médica Superior. 2009; 23(3): 45-62.

16. Organización Panamericana de la Salud/ Organización Mundial de la Salud. Servicios farmacéuticos basados en la atención primaria de salud. Documento de posición de la OPS/OMS. Washington, DC: OPS. Serie La renovación de la atención primaria de salud en las Américas. 2013. 6: $1: 57$.

17. Camarena B, Velarde D. Educación superior y mercado laboral: vinculación y pertinencia social ¿Por qué? y ¿Para qué?. Estud. Soc. 2009. 17: 106125. 